\title{
State-dependent M/G/1 type queueing analysis for congestion control in data networks
}

\author{
Eitan Altman, Kostya Avrachenkov, Chadi Barakat ${ }^{\dagger}$, Rudesindo Núñez-Queija
}

\begin{abstract}
We study in this paper a TCP-like linear-increase multiplicative-decrease flow control mechanism. We consider congestion signals that arrive in batches according to a Poisson process. We focus on the case when the transmission rate cannot exceed a certain maximum value. We write the Kolmogorov equations and we use Laplace Transforms to calculate the distribution of the transmission rate in the steady state as well as its moments. Our model is particularly useful to study the behavior of TCP, the congestion control mechanism in the Internet. By a simple transformation, the problem can be reformulated in terms of an equivalent $M / G / 1$ queue, where the transmission rate in the original model corresponds to the workload in the 'dual' queue. The service times in the queueing model are not i.i.d., and they depend on the workload in the system.
\end{abstract}

Keywords - TCP congestion control, batch Poisson process, Kolmogorov equation, Laplace Transform.

\section{INTRODUCTION}

$\mathrm{I}^{\mathrm{N}}$ $\mathrm{N}$ today's high speed telecommunication networks, a large 1 part of the traffic is able to adapt its rate to the congestion conditions of the network. Congestion control is typically designed so as to allow the transmission rate to increase linearly in time in the absence of congestion signals, whereas when congestion is detected, the rate decreases by a multiplicative factor. This is both the case of the Available Bit Rate (ABR) service category in ATM [1] (see definition and use of RDF and RIF) as well as the Transmission Control Protocol (TCP) in the Internet environment [10], [22]. Congestion is detected by the source through signals. In case of ABR, the congestion signals are RM (Resource Management) cells that have been marked due to congestion information in some switch along the path of the connection. In case of the Internet, the congestion signals are packet losses that are detected by the source either through the expiration of a retransmission timer, or through some negative acknowledgement mechanism (three duplicate ACKs [22]). There is also a proposal to add some explicit congestion signaling to the Internet (the ECN proposal [7]).

The performance evaluation of congestion control mechanisms is an important issue for network and protocol design. This evaluation requires a description of times between the arrivals of consecutive congestion signals. Experimentations over the Internet [4], [15] have shown that on long distance connections, the Poisson assumption about the times between congestion signals is quite reasonable. This happens when the throughput of the studied connection is small compared to the exogenous traffic, and when the number of hops on the path is large so that the superposition of the packet drops in routers leads to exponential times between congestion signals. For local area networks, we noticed that the congestion signals may arrive in

E. Altman, K. Avrachenkov, and C. Barakat are with INRIA, SophiaAntopolis, France. Email: \{altman,kavratch,cbarakat\}@sophia.inria.fr.

R. Núñez-Queija is with CWI, Amsterdam, The Netherlands. Email: sindo@cwi.nl.

$\dagger$ Corresponding author. bursts [4]. However, the times between bursts correspond well to the Poisson assumption. For this reason, we consider the case when congestion signals arrive in batches according to a Poisson process. Batches contain a random number of congestion signals and each such signal causes the division of the transmission rate by some constant $\gamma$. In the sequel, we also refer to a batch of congestion signals as a loss event.

We focus on the case when a certain limitation on the transmission rate exists. We determine the exact expression of the throughput under such a limitation. In the literature, only simplistic approximations have been proposed [3], [19] so far. We study two possible scenarios that lead to such a limitation:

(i) Peak Rate limitation: the limitation is not due to congestion in the network but rather to some external agreement. In that case, when the transmission rate reaches a certain level $M$, it remains constant until a loss event appears. For example in case of TCP, the window cannot exceed the buffer space available at the receiver [22]. This is known as the end-to-end flow control of TCP. In the ABR service of ATM, the transmission rate cannot exceed the Peak Cell Rate imposed by the contract between the user and the network.

(ii) Congestion limitation: the limitation on the transmission rate is due to congestion in the network that occurs whenever the input rate reaches a level $M$. In that case we shall have an extra batch of congestion signals when the level $M$ is attained which also causes a reduction of the transmission rate by a random factor. A typical example of such limitation is the available capacity in the network. There is a maximum limit on the number of packets a source can keep in the network and once this limits is exceeded packets are dropped. Another example is the reserved bandwidth in a Differentiated Services network [9] in cases when packets exceeding the reserved bandwidth are dropped rather than injected into the network as low priority packets [21].

In the particular case in which the batches contain a single congestion signal, the peak rate limitation model reduces to the one studied in [15], who already attempted at computing the first two moments of the transmission rate. A remarkable observation is done in that reference showing that the flow control can be reformulated in terms of an equivalent M/G/1 queue, where the transmission rate is translated into the workload of the queue. The congestion signals correspond to customers arriving at the queue according to a Poisson process. The service times in the 'dual' queueing model are not i.i.d., and they depend on the workload in the system. This transformation is also valid in our more general setting except that in our model with congestion limitation, there is an additional arrival in the equivalent queueing model (in addition to the Poisson arrival stream) that occurs whenever the queue empties. Another difference is that in our 
case congestion signals arrive in batches. Without loss of generality, we consider that the occurrence of a batch of congestion signals corresponds to the arrival of a client into the dual queueing model. Hence, the service time of a client is also a function of the number of congestion signals in the corresponding batch.

We solve in this paper the Kolmogorov equations and obtain the exact probability distribution as well as the moments of the transmission rate (of the window in case of TCP) for both problems. In doing so, we correct an error ${ }^{1}$ in [15].

We briefly mention some related results. Queueing analysis with service times that depend on the workload or on the queue length have been also considered in [2], [12], [17], [20]. Our model is a special case of the one studied in [20], where an implicit characterization of the steady state distribution is obtained (closed-form expressions were obtained for special cases that do not cover our model). In [12] an asymptotic approximation is used for solving state-dependent GI/G/1 queues in which both inter-arrival times, service requirements and the service rate may depend on the workload. The peak rate limitation model is a special case of the model with a general stationary and ergodic arrival process studied in [4]. For that model only bounds on the throughput were obtained. Exact expressions for the throughput were obtained there for the case in which no limitation on the transmission rate exists (see also [3], [14], [16], [19]).

The structure of the paper is as follows. In Section II we describe a general model of flow control with limitation on the transmission rate and we provide a preliminary analysis. The two cases of peak rate limitation and congestion limitation are described separately in Sections II-A and II-B. It is shown that a special case of the model with congestion limitation reduces to that of the model with peak rate limitation. Hence, it suffices to analyze the latter case. We then derive the moments and the distribution of the transmission rate in Sections III and IV in terms of one unknown constant: the probability of attaining the maximum rate. This constant is computed in Section V. In Section VI we specify our model to an important particular case, that of one congestion signal per batch and a reduction factor equal to one half. Based on this particular case, we present in Section VII some numerical and experimental results to validate the model. We conclude the paper with a section on future research.

\section{Flow CONTROL WITH RATE LimitATION: MODELS AND PRELIMINARY ANALYSIS}

We present in this section our model for the rate evolution of the flow control mechanism. In the sequel we adopt the usual terminology for TCP, the well known window-based congestion control protocol of the Internet: we shall work with the window size rather than the transmission rate. The transmission rate of a window-based flow control mechanism is at any moment equal to the window size divided by the round-trip time (RTT) of the connection.

Let $M$ denote the maximum window size. The limitation on the window size is either due to a peak rate limitation or to a congestion limitation. In the following we explain the similarities and the differences between the models in the two cases. While

\footnotetext{
${ }^{1}$ In a private communication, the authors of [15] announced to replace the draft with a new one
}

no congestion signal is received and the window is smaller than $M$, the window of the protocol increases linearly at rate $\alpha>0$. In case of TCP, $\alpha=1 /(b \cdot R T T)$ where $b$ is the number of data packets covered by an ACK (usually 2, see e.g.[19], [22]) and where $R T T$ is the average round-trip time of the connection assumed to be independent of the window size [4], [19].

We assume that batches containing a random number of congestion signals arrive according to an independent Poisson process. We denote the sizes (i.e., the numbers of congestion signals) of consecutive batches by $N_{1}, N_{2}, N_{3}, \ldots$, and we assume that these constitute an i.i.d. sequence. The size of an arbitrary batch is generically denoted by $N \stackrel{d}{=} N_{k}$. The Poisson process and the sequence $N_{k}, k=1,2, \ldots$, are independent of each other and independent of the past evolution of the window. For each congestion signal received, the window is divided by a factor $\gamma>1$ which is a fixed parameter. That is, if an arriving batch contains $N=n$ congestion signals, the window is multiplicatively decreased by a factor $\gamma^{-n}$. Immediately after the multiplicative decrease, the window restarts its linear increase. In case of peak rate limitation, the window stays constant at $M$ when this maximum level is reached until the next congestion signal is received. In case of congestion limitation, immediately upon reaching $M$, a congestion signal is received and the window is decreased. We present the two cases separately in Sections II-A and II-B.

Before doing so, we introduce some further common notation. We denote the p.g.f. (probability generating function) of the distribution of $N$ by

$$
Q(z):=\mathrm{E}\left[z^{N}\right]=: \sum_{n=1}^{\infty} z^{n} q_{n}, \quad|z| \leq 1
$$

Note that the peak rate limitation model with $\gamma=2$ and $q_{1}=1$ reduces to the model studied in [15], where congestion signals appear according to a Poisson process and where the window is divided by two upon every congestion signal occurrence. By considering a general model, we aim to account for a wide range of flow control mechanisms other than TCP and for future enhancements to TCP congestion control.

Let us denote the window size at time $t \geq 0$ by $W(t) \in$ $(0, M]$. We have the following stability result which follows from Theorem 1 in [4]:

Theorem II.1: There exists a stationary process $W^{*}(t)$ such that $W(t)$ converges to $W^{*}(t)$ in distribution for any initial state. Moreover, we have P-a.s.

$$
\lim _{t \rightarrow \infty} \sup _{s \geq t}\left|W(s)-W^{*}(s)\right|=0
$$

Note that (2) implies that the stationary distribution of $W(t)$ is unique. For $x \in(0, M]$, denote the (time-average) distribution function by

$$
F(x):=\lim _{T \rightarrow \infty} \frac{1}{T} \int_{t=0}^{T} \mathbf{P}\{W(t) \leq x\} \mathrm{d} t .
$$

It follows from Theorem II. 1 that this limit is independent of $W(0)$ and coincides with the stationary distribution of $W(t)$. 
We first assume that $F(x)$ is continuous in $x \in(0, M)$ (in the case of peak rate limitation it is clear from physical considerations that $F(x)$ has an atom at $x=M)$. Under this assumption we find a function $F(x)$ which is an equilibrium distribution for the window size and, hence, from its uniqueness it follows that it is the desired distribution. Instead of $F(x)$ it will be convenient to work with the complementary distribution function

$$
\bar{F}(x)=1-F(x)=\mathbf{P}\{W>x\}, \quad x \in(0, M] .
$$

To differentiate between the cases of peak rate limitation and congestion limitation, in the latter case we attach a superscript ${ }^{\mathrm{cl}}$ to the symbols introduced above, e.g., the distribution function is denoted by $F^{\mathrm{cl}}(x)$. Next we treat the two cases separately. In particular we show how the analysis of the model with congestion limitation reduces to that of the model with peak rate limitation. Therefore, we concentrate on the peak rate limitation model in the subsequent sections.

\section{A. Flow control with peak rate limitation}

With peak rate limitation, when the window reaches the maximum level $M$, it stays there until the next congestion signal is received. In Section II-C below we show that the window size process $W(t)$ can be related to the workload of an M/G/1 queue (see also [15]). The workload of this state-dependent M/G/1 queue can be seen to be a Markov process (e.g. see [11]), and hence the window size evolution $W(t)$ is a Markov process as well. With this in mind, we derive a steady-state Kolmogorov equation for $\bar{F}(x)=\mathbf{P}\{W>x\}$ which will be the basis to our analysis. We use the following up and down crossing argument: Assume that the process is in equilibrium and consider a level $x \in(0, M)$. Whenever the window size increases from less than or equal to $x$ to more than $x$ we say that an up crossing of the level $x$ has occurred. Similarly, if the window size decreases from more than $x$ to less than or equal to $x$ we say that a down crossing of the level $x$ has occurred. Let $[t, t+\Delta]$ be a small time interval, where $t$ is a deterministic time moment. When the process is in equilibrium, the probability of up-crossing

$$
(1-\lambda \Delta) \mathbf{P}\{x-\alpha \Delta<W \leq x\}+\mathrm{o}(\Delta)
$$

is equal to the probability of down-crossing

$$
\lambda \Delta \sum_{n=1}^{\infty} q_{n} \mathbf{P}\left\{x<W \leq \min \left(\gamma^{n} x, M\right)\right\}+\mathrm{o}(\Delta) .
$$

After equating these, we pass $\Delta \downarrow 0$. Since we assumed that $F(x)=\mathbf{P}\{W \leq x\}$ is continuous for $x<M$ (see Remark 1 for a justification of this assumption), we conclude that the derivative of $F(x)$ exists and is continuous for all $x$ except at $x=M \gamma^{-n}$, when $q_{n}>0$. For $x \in(0, M) \backslash\left\{M \gamma^{-n}\right\}_{n=1,2, \ldots}$ we obtain the following steady-state Kolmogorov equation

$$
\alpha \frac{\mathrm{d}}{\mathrm{d} x} \mathbf{P}\{W \leq x\}=\lambda \sum_{n=1}^{\infty} q_{n} \mathbf{P}\left\{x<W \leq \min \left(\gamma^{n} x, M\right)\right\},
$$

or, equivalently,

$$
-\alpha \frac{\mathrm{d}}{\mathrm{d} x} \bar{F}(x)=\lambda\left(\bar{F}(x)-\sum_{n=1}^{\infty} q_{n} \bar{F}\left(\min \left(\gamma^{n} x, M\right)\right)\right) .
$$

From this differential equation we shall determine $\bar{F}(x), x \in$ $(0, M)$, in terms of the probability

$$
P_{M}:=\mathbf{P}\{W=M\}=1-F(M-)=\bar{F}(M-) .
$$

In Section III we first use (4) to determine the moments of the window size distribution in terms of $P_{M}$. Then we find the distribution function itself in Section IV. The unknown $P_{M}$ is then determined using the fact that $\bar{F}(x)$ is a complementary probability distribution function $(\bar{F}(0)=1)$. However, the expression obtained for $P_{M}$ in this way, does not lend itself for computational purposes. Therefore we show an elegant alternative to determine $P_{M}$ in Section $\mathrm{V}$, which leads to an efficient and numerically stable algorithm for computations.

\section{B. Flow control with congestion limitation}

When the maximum window size $M$ is due to congestion limitation, immediately upon reaching the level $M$ a batch of congestion signals is generated. Here we study the case when the size of such a batch has the same distribution as the random variable $N$. In [5] we treat a more general case when the number of congestion signals that result from reaching $M$ has a different distribution than $N$. Similarly as in Section II-A, we can derive the following differential equation for $\bar{F}^{\mathrm{cl}}(x), 0<x<M$ :

$$
\begin{aligned}
-\alpha \frac{\mathrm{d}}{\mathrm{d} x} \bar{F}^{\mathrm{cl}}(x)=\lambda & \left(\bar{F}^{\mathrm{cl}}(x)-\sum_{n=1}^{\infty} q_{n} \bar{F}^{\mathrm{cl}}\left(\min \left(\gamma^{n} x, M\right)\right)\right) \\
& +\lambda g \mathbf{P}\left\{N \geq \frac{\ln (M)-\ln (x)}{\ln (\gamma)}\right\},
\end{aligned}
$$

with,

$$
g:=-\left.\frac{\alpha}{\lambda} \frac{\mathrm{d}}{\mathrm{d} y} \bar{F}^{\mathrm{cl}}(y)\right|_{y=M-} .
$$

The additional term, compared to (4), comes from the fact that a down crossing of the level $x$ may be due to the fact that the level $M$ is reached and that the rate is decreased by a factor $\gamma^{-n}$ with $\gamma^{-n} M \leq x$. Note that if $\bar{F}(x)$ is the unique complementary distribution function satisfying (4) then

$$
\bar{F}^{\mathrm{cl}}(x):=\frac{\bar{F}(x)-P_{M}}{1-P_{M}}, \quad 0<x<M,
$$

is the unique complementary distribution function satisfying (5). This follows immediately by substituting (6) into (5). This relation has a simple geometric interpretation. Using the fact that the Poisson process is memoryless, if we consider the model with peak rate limitation only at moments when the window is less than $M$ (i.e., we cut out all periods where the window equals $M)$, what we get is identical to the model with congestion limitation. Thus, we can concentrate on finding the distribution function $F(x)$ for the peak rate limitation model and then use (6) or the equivalent:

$$
F^{\mathrm{cl}}(x)=\frac{F(x)}{1-P_{M}} .
$$

In particular, the moments of the window size in the two models are related by:

$$
\mathrm{E}\left[\left(W^{\mathrm{cl}}\right)^{k}\right]=\frac{\mathrm{E}\left[W^{k}\right]-P_{M} M^{k}}{1-P_{M}} .
$$


In Section III below we derive a recursive relation for $\mathrm{E}\left[W^{k}\right]$. Combined with (7), this gives a recursion on $\mathrm{E}\left[\left(W^{\mathrm{cl}}\right)^{k}\right]$ which we report at this point for completeness:

$$
\mathrm{E}\left[\left(W^{\mathrm{cl}}\right)^{k}\right]=\frac{k \alpha \mathrm{E}\left[\left(W^{\mathrm{cl}}\right)^{k-1}\right]}{\lambda\left(1-Q\left(\gamma^{-k}\right)\right)}-\frac{P_{M}}{1-P_{M}} M^{k} .
$$

Remark 1: We emphasize that in the congestion limitation model, the quantity $P_{M}$ has no clear interpretation. In Section $\mathrm{V}$ we use the interpretation of this quantity in the peak rate limitation model to compute it. If we were to analyze the congestion limitation model without using (6), then from (5) we could express $\bar{F}^{\mathrm{cl}}(x)$ - using the same techniques as in Section IV in terms of $g$ instead of $P_{M}$. Note that these two constants are related:

$$
g=\frac{P_{M}}{1-P_{M}}
$$

The constant $g$ can be determined using that $\bar{F}^{\text {cl }}(x)$ is a complementary probability distribution, see (24) below. Since from the analysis of Section $\mathrm{V}$ we obtain a more tractable expression for $P_{M}$ (see Remark 2 for a related discussion), we will not further dwell on this approach.

\section{The dual $M / G / 1$ queueing model}

Before proceeding with determining the moments and the distribution of the window size, we briefly show how the flow control problem can be related to an $\mathrm{M} / \mathrm{G} / 1$ queueing problem with service depending on the system workload, see also [15]. First we concentrate on peak rate limitation, then we comment on congestion limitation. Define

$$
U(t)=\frac{M-W(t)}{\alpha}
$$

Namely, $U(t)$ is obtained by 'flipping' $W(t)$ around a horizontal line. In particular, the area between $W(t)$ and the maximum window size $M$ (Figure 1) corresponds to the area below $U(t)$. Note that $U(t)$ resembles the evolution in time of the workload (or the virtual waiting time) in a queueing system. A window equal to $M$ corresponds to an empty queueing system. The linear increase in workload between arrivals of congestion signals corresponds to the decrease in workload due to service in the $\mathrm{M} / \mathrm{G} / 1$ model. The arrival of a batch of congestion signals in our model corresponds to an arrival in the M/G/1 model. The reduction of the window upon a loss event corresponds to the increase in workload upon arrival in the equivalent M/G/1 model. Given that the amount by which the window is reduced depends on the current value of the window (and of course on the number of congestion signals in the batch), the service time in the dual queueing model is dependent on the current workload there. We conclude that the dual model behaves indeed as an M/G/1 queue (infinite buffer capacity, one server and Poisson arrivals with intensity $\lambda$ ) with state-dependent service requirements. If $U_{n}$ is the workload seen by arrival $n$ in the M/G/1 queue, then its service time $x_{n}$ is equal to

$$
x_{n}=\left(\frac{M}{\alpha}-U_{n}\right) \cdot\left(1-\frac{1}{\gamma^{N_{n}}}\right),
$$

where $N_{n}$ is the number of congestion signals in the $n$th batch of congestion signals in the original model. Instead of working with the window as we are doing in this paper, one could solve the queueing problem and switch back to the flow control problem by using Equation (10). In particular, $\mathrm{E}\left[W^{k}\right]=$ $\mathrm{E}\left[(M-\alpha U)^{k}\right], \mathbf{P}\{W \leq x\}=1-\mathbf{P}\{U \leq(M-x) / \alpha\}$ for $x<M$ and $P_{M}$ is equal to the fraction of time the server in the dual queueing model is idle.

In the case of congestion limitation, the only difference in the dual queueing model is that we have an additional arrival once the system becomes empty. This makes of the arrival process the sum of a Poisson process of intensity $\lambda$ and another process that depends on the workload of the system. The definition of the service time and the transformation back to the flow control problem remain the same.

\section{MOMEnTS OF THE WINDOW SIZE DISTRIBUTION}

We further focus on the model with peak rate limitation. In this section we study the moments of the window size. The $k$-th moment of the transmission rate can be simply obtained by dividing the $k$-th moment of the window size by $(R T T)^{k}$. Of particular interest is the expectation of the transmission rate which coincides with the throughput of the transfer or the time average of the transmission rate. Let $\bar{X}$ denote the throughput. We have

$$
\bar{X}=\lim _{T \rightarrow \infty} \frac{1}{T} \int_{0}^{T} X(t) d t=\frac{\mathrm{E}[W]}{R T T} .
$$

The second moment is also important since it tells us how much the transmission rate varies. Some applications (e.g., real time multimedia flows) are sensitive to variations in transmission rate. One could envisage to tune the flow control so that to reduce these variations.

Define for $\operatorname{Re}(\omega) \geq 0$ the LST (Laplace-Stieltjes Transform) of the window size distribution by

$$
\hat{f}(\omega)=\int_{x=0}^{M+} \mathrm{e}^{-\omega x} \mathrm{~d} F(x) .
$$

Taking LTs (Laplace Transforms) in (4) leads to:

$$
\begin{aligned}
\alpha\left(\hat{f}(\omega)-P_{M} \mathrm{e}^{-\omega M}\right)= & \lambda \frac{1-\hat{f}(\omega)}{\omega} \\
& -\lambda \sum_{n=1}^{\infty} \gamma^{-n} q_{n} \frac{1-\hat{f}\left(\gamma^{-n} \omega\right)}{\gamma^{-n} \omega} .
\end{aligned}
$$

Note that (12) holds in particular for $M=\infty$, i.e., no limitation on the window size, in which case $P_{M}=0$. Using $\mathrm{E}\left[W^{k}\right] \leq$ $M^{k}, k=1,2, \ldots$, we may write

$$
\begin{aligned}
\hat{f}(\omega) & =1+\sum_{k=1}^{\infty} \frac{(-\omega)^{k}}{k !} \mathrm{E}\left[W^{k}\right], \\
\frac{1-\hat{f}\left(\gamma^{-n} \omega\right)}{\gamma^{-n} \omega} & =\sum_{k=0}^{\infty} \frac{\left(-\gamma^{-n} \omega\right)^{k}}{(k+1) !} \mathrm{E}\left[W^{k+1}\right] .
\end{aligned}
$$

Substituting this in (12), using the absolute convergence of the doubly-infinite series to interchange the order of summation and 


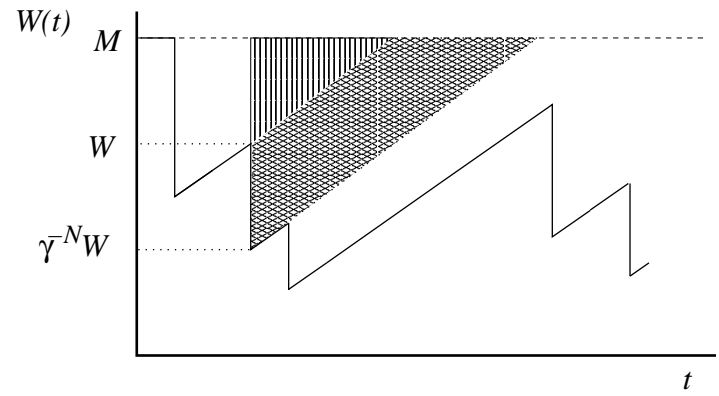

Fig. 1. Area associated with a single loss

equating the coefficients of equal powers of $\omega$ we get, for $k=$ $1,2, \ldots$,

$$
\mathrm{E}\left[W^{k}\right]=\frac{k \alpha\left(\mathrm{E}\left[W^{k-1}\right]-P_{M} M^{k-1}\right)}{\lambda\left(1-Q\left(\gamma^{-k}\right)\right)},
$$

from which the moments of the window size distribution can be obtained recursively. In particular we find for $k=1,2$ :

$$
\begin{aligned}
\mathrm{E}[W] & =\frac{\alpha\left(1-P_{M}\right)}{\lambda\left(1-Q\left(\gamma^{-1}\right)\right)} \\
\mathrm{E}\left[W^{2}\right] & =\frac{2 \alpha\left[\alpha\left(1-P_{M}\right)-\lambda P_{M} M\left(1-Q\left(\gamma^{-1}\right)\right)\right]}{\lambda^{2}\left(1-Q\left(\gamma^{-1}\right)\right)\left(1-Q\left(\gamma^{-2}\right)\right)}
\end{aligned}
$$

These first two moments can be also obtained using direct arguments, see Remarks 1 and 2 below. Such arguments were also used by Misra et al. [15] for the case $\gamma=2$ and $N \equiv 1$. However, in their analysis an error appears which results in an additional equation besides (14) and (15) from which they determine an erroneous expression for the probability $P_{M}$ (see Remark 2).

Remark 1: The mean window size can be obtained by considering the mean drift. The upward drift of the window size is given by $\alpha \mathbf{P}\{W<M\}$ and the downward drift equals $\lambda \mathrm{E}[W]\left(1-\mathrm{E}\left[\gamma^{-N}\right]\right)$. Equating these gives (14).

We can further derive $\mathrm{E}\left[W^{2}\right]$ applying an argument similar to Little's law as was done by Misra et al. [15] for the case $\gamma=2$ and $N \equiv 1$. For details we refer to [5]. The main idea is sketched in the following. For the dual queueing model described in Section II-C, we can equate the mean workload $\mathrm{E}[U]$ with $\lambda$ times the mean area below $U(t)$ 'induced by a single arrival' (use that Poisson Arrivals See Time Averages). Back in the original model, the 'mean surface' of the area above $W(t)$ in Figure 1 equals $M-\mathrm{E}[W]$.

The expected surface of the area 'induced' by a single loss event (the surface of the larger triangle minus that of the smaller one) is equal to

$$
\frac{1}{2 \alpha}\left(\left(Q\left(\gamma^{-2}\right)-1\right) \mathrm{E}\left[W^{2}\right]-2 M\left(Q\left(\gamma^{-1}\right)-1\right) \mathrm{E}[W]\right) .
$$

Multiplying this by $\lambda$, equating the result with $M-\mathrm{E}[W]$ and using (14) indeed gives (15).

Remark 2: For a special case of our model, yet another way is pursuit in [15] to derive (14) and (15). However, there, the final result is incorrect due to a small error in an intermediate step. Defining $P_{M}(t):=\mathbf{P}\{W(t)=M\}, \mathrm{E}[W(t)]$ and $\mathrm{E}\left[W(t)^{2}\right]$ satisfy:

$$
\frac{\mathrm{d}}{\mathrm{d} t} \mathrm{E}[W(t)]=-\lambda\left(1-Q\left(\gamma^{-1}\right)\right) \mathrm{E}[W(t)]
$$

$$
\begin{array}{ll} 
& +\alpha\left(1-P_{M}(t)\right), \\
\frac{\mathrm{d}}{\mathrm{d} t} \mathrm{E}\left[W(t)^{2}\right]= & -\lambda\left(1-Q\left(\gamma^{-2}\right)\right) \mathrm{E}\left[W(t)^{2}\right] \\
& +2 \alpha\left(\mathrm{E}[W(t)]-M P_{M}(t)\right) .
\end{array}
$$

In steady state we have $\mathrm{E}[W(t)] \equiv \mathrm{E}[W], \mathrm{E}\left[W(t)^{2}\right] \equiv$ $\mathrm{E}\left[W^{2}\right]$ and $P_{M}(t) \equiv P_{M}$. Substitution into the above gives (14) together with

$$
0=-\lambda\left(1-Q\left(\gamma^{-2}\right)\right) \mathrm{E}\left[W^{2}\right]+2 \alpha\left(\mathrm{E}[W]-P_{M} M\right) .
$$

Together with (14) this leads to (15). For the case $\gamma=2$ and $N \equiv 1$, the formula given in [15] for $\mathrm{E}\left[W^{2}\right]$ (below Formula (4) in that reference) differs from (16) by a factor $-\alpha=-1 / R T T$. This resulted in a third (incorrect) equation which is linearly independent of (14) and (15) from which $P_{M}$ was determined simultaneously with $\mathrm{E}[W]$ and $\mathrm{E}\left[W^{2}\right]$. In Section $\mathrm{V}$ we show how $P_{M}$ can be determined correctly and computed efficiently.

\section{WINDOW SIZE DISTRIBUTION FUNCTION}

We find in this section the explicit expression of the cumulative distribution of the window size. The distribution of the transmission rate can be simply obtained by rescaling the window axis by $1 /$ RTT. We start first by the case of finite $M$. We provide the expression of the distribution in every interval $\left[M / \gamma^{k}, M / \gamma^{k-1}\right]$ with $k=1,2, \ldots$. Then, for the case $M=\infty$, we give an expression of the distribution for any $x>0$ as an infinite sum of exponentials.

\section{A. Window distribution for finite $M$}

For $M / \gamma \leq x<M$, Equation (4) reduces to:

$$
-\alpha \frac{\mathrm{d}}{\mathrm{d} x} \bar{F}(x)=\lambda \bar{F}(x),
$$

hence,

$$
\bar{F}(x)=P_{M} \mathrm{e}^{\frac{\lambda}{\alpha}(M-x)}, \quad \frac{M}{\gamma} \leq x<M .
$$

To find the entire distribution we introduce, for $k=1,2,3, \ldots$,

$$
\bar{F}_{k}(x):=\bar{F}(x), \quad \frac{M}{\gamma^{k}} \leq x<\frac{M}{\gamma^{k-1}} .
$$

Equation (4) can now be written as:

$$
\frac{\mathrm{d}}{\mathrm{d} x} \bar{F}_{k}(x)=-\frac{\lambda}{\alpha} \bar{F}_{k}(x)+\frac{\lambda}{\alpha} \sum_{n=1}^{k-1} q_{n} \bar{F}_{k-n}\left(\gamma^{n} x\right) .
$$

Since $\bar{F}(x)$ is continuous for $0<x<M$ we have:

$$
\bar{F}_{k}\left(\frac{M}{\gamma^{k-1}}\right)=\bar{F}_{k-1}\left(\frac{M}{\gamma^{k-1}}\right), \quad k=2,3, \ldots
$$

$\bar{F}_{k}$ is recursively given by

$$
\begin{aligned}
\bar{F}_{k}(x)= & \bar{F}_{k-1}\left(M / \gamma^{k-1}\right) \mathrm{e}^{\frac{\lambda}{\alpha}\left(\frac{M}{\gamma^{k-1}}-x\right)} \\
& -\frac{\lambda}{\alpha} \mathrm{e}^{-\frac{\lambda}{\alpha} x} \int_{u=x}^{M / \gamma^{k-1}} \mathrm{e}^{\frac{\lambda}{\alpha} u} \sum_{n=1}^{k-1} q_{n} \bar{F}_{k-n}\left(\gamma^{n} u\right) \mathrm{d} u .
\end{aligned}
$$


We conclude from the above recursion that a solution to (19) and (20) has the following form

$$
\bar{F}_{k}(x)=P_{M} \sum_{i=1}^{k} c_{i}^{(k)} \mathrm{e}^{-\frac{\lambda}{\alpha} \gamma^{i-1} x}, \quad k=1,2, \ldots
$$

To determine the coefficients $c_{i}^{(k)}$, we substitute (21) into (19). Then, equating terms with the same exponents, we get the following recursive formula

$$
c_{i+1}^{(k)}=\frac{1}{1-\gamma^{i}} \sum_{n=1}^{i} q_{n} c_{i-n+1}^{(k-n)}, \quad i=1, \ldots, k-1 .
$$

Once the coefficients $c_{i}^{(k)}, i=2, \ldots, k$ are computed, the coefficient $c_{1}^{(k)}$ can be determined from (20):

$$
c_{1}^{(k)}=e^{\frac{\lambda}{\alpha} \frac{M}{\gamma^{k-1}}}\left[\sum_{i=1}^{k-1} c_{i}^{(k-1)} e^{-\frac{\lambda}{\alpha} \gamma^{i-k} M}-\sum_{i=2}^{k} c_{i}^{(k)} e^{-\frac{\lambda}{\alpha} \gamma^{i-k} M}\right] .
$$

Note that to compute the coefficients $c_{i}^{(k)}$, we do not need $P_{M}$. Hence, using that $\bar{F}(x)$ is a complementary distribution function, $P_{M}$ is then determined by:

$$
P_{M}\left(\lim _{k \rightarrow \infty} \sum_{i=1}^{k} c_{i}^{(k)} \mathrm{e}^{-\frac{\lambda}{\alpha} M / \gamma^{k-i}}\right)=\lim _{k \rightarrow \infty} \bar{F}_{k}\left(\frac{M}{\gamma^{k-1}}\right)=1
$$

However, this relation is not suitable to compute $P_{M}$, see Remark 2 below.

Remark 1: With (18) and (21) we have found an equilibrium distribution function $F(x)$ satisfying (4). By Theorem II.1 it is the unique solution and, hence, the assumption that $F(x)$ is continuous for $x<M$ is justified.

Remark 2: Recursion (22) is suitable to determine the distribution function on an interval $M / \gamma^{k} \leq x \leq M$ when $k$ is not too large. For large $k$ the recursion may become instable, since it involves subtraction of numbers of the same order. Therefore (24) is not suitable to compute $P_{M}$. In Section $\mathrm{V}$ below we derive an alternative expression for $P_{M}$, which leads to a numerically stable and efficient algorithm to compute $P_{M}$.

\section{B. Window distribution for infinite $M$}

In this case, the results derived in the previous subsection cannot be applied immediately by letting $M$ go to infinity. However, we can derive the LST of the window size distribution by similar arguments. When $M=\infty$, (12) becomes

$$
\hat{f}(\omega)=-\frac{\lambda}{\alpha}\left[\frac{\hat{f}(\omega)}{\omega}-\sum_{n=1}^{\infty} q_{n} \frac{\hat{f}\left(\gamma^{-n} \omega\right)}{\omega}\right],
$$

or, equivalently,

$$
\hat{f}(\omega)=\frac{\frac{\lambda}{\alpha}}{\omega+\frac{\lambda}{\alpha}} \sum_{n=1}^{\infty} q_{n} \hat{f}\left(\gamma^{-n} \omega\right) .
$$

Substituting the above equation repeatedly into itself $l$ times, applying partial fraction expansion at each step, and then taking $l \rightarrow \infty$, we conclude that $\hat{f}(\omega)$ can be expressed as follows:

$$
\hat{f}(\omega)=\sum_{i=0}^{\infty} c_{i} \frac{-\frac{\lambda}{\alpha} \gamma^{i}}{\omega+\frac{\lambda}{\alpha} \gamma^{i}}
$$

for certain coefficients $c_{i}$ (this is formally justified later). To determine the constants $c_{i}, i=0,1, \ldots$, we substitute (26) into (25) and equate coefficients multiplying the terms $1 /\left(\omega+\frac{\lambda}{\alpha} \gamma^{i}\right)$. This leads to the recursive formula

$$
\frac{c_{i}}{c_{0}}=\frac{1}{1-\gamma^{i}} \sum_{k=1}^{i} q_{k} \frac{c_{i-k}}{c_{0}}
$$

which determines the ratios $c_{i} / c_{0}$ (it is for this reason that both sides contain a factor $1 / c_{0}$ ). The coefficient $c_{0}$ follows from $\hat{f}(0)=-\sum_{i=0}^{\infty} c_{i}=1$ :

$$
c_{0}=-\left(1+\sum_{i=1}^{\infty} \frac{c_{i}}{c_{0}}\right)^{-1} .
$$

It can be shown that the infinite series in the expression of $c_{0}$ converges and hence all the coefficients $c_{i}$ exist. The proof of convergence is given in Appendix A. The inversion of (26) back into the time domain gives:

$$
F(x)=C+\sum_{i=0}^{\infty} c_{i} \mathrm{e}^{-\frac{\lambda}{\alpha} \gamma^{i} x},
$$

with $C=1$ (because $F(0)=0$ ). Given that the coefficients $c_{i}$ exist, the above series is absolutely convergent for any value of $x \in[0, \infty)$. Thus, it is the unique solution to (4) when $M=\infty$. For the case of no window size limitation and $N \equiv 1,\left(q_{1}=1\right)$, $F(x)$ was already obtained in [18].

\section{THE PROBABILITY OF MAXIMUM WINDOW SIZE}

In Sections III and IV we determined the window size distribution and its moments in terms of $P_{M}$. In this section we derive an expression for $P_{M}$ from which it can be computed efficiently. For this we introduce the random variable $T(x)$ which is the time until the window size returns to the value $x$, starting just after a loss event occurs with the window size being equal to $x \in(0, M]$. We denote its expectation by $E(x):=\mathrm{E}[T(x)]$, $x \in(0, M]$. Then, from elementary renewal theory,

$$
P_{M}:=\mathbf{P}\{W=M\}=\frac{1 / \lambda}{1 / \lambda+E(M)} .
$$

We now proceed to find the function $E(x)$. A typical evolution of the window size is depicted in Figure 2. For simplicity in the figure only loss events having $N=1$ are depicted. The times to recover from losses are partly cut out of the picture (denoted by the shaded areas).

Suppose for the moment that the initial loss (at the level $x$ ) was such that $N=n$ (in the figure $n=1$ ). Let $T_{n}(x)$ be the time to get back at level $x$ conditional on $N=n$ and we further write $E_{n}(x):=\mathrm{E}\left[T_{n}(x)\right]:=\mathrm{E}[T(x) \mid N=n]$. Note that

$$
E(x)=\sum_{n=1}^{\infty} q_{n} E_{n}(x) .
$$




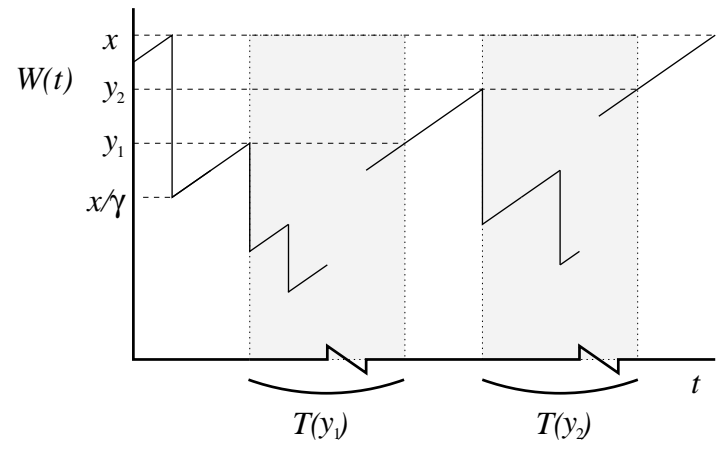

Fig. 2. TCP window

If no losses occur during the time $T_{n}(x)$ then $T_{n}(x)=(1-$ $\left.\gamma^{-n}\right) x / \alpha$, i.e., the window size $x$ is reached in a straight line from the starting point at $\gamma^{-n} x$ (in the figure $\gamma^{-1} x$ ). Each time a loss occurs at a level $y \in\left(\gamma^{-n} x, x\right)$ it takes $T(y)$ time units to get back at the level $y$. Because of the memoryless property of the Poisson process, if we take out the shaded areas in Figure 2 and concatenate the non-shaded areas then the cut points (where the shaded areas used to be) form a Poisson process on the straight line from $\gamma^{-n} x$ to $x$. Thus if the cut points are given by $y_{1}, y_{2}, \ldots, y_{m}$ (in the figure $m=2$ ) then

$$
E_{n}(x)=\frac{\left(1-\gamma^{-n}\right) x}{\alpha}+E\left(y_{1}\right)+E\left(y_{2}\right)+\ldots+E\left(y_{m}\right) .
$$

Since the loss process is a Poisson process, the mean number of cut points is $\lambda\left(1-\gamma^{-n}\right) x / \alpha$ and the position of each of the points $y_{j}$ is uniformly distributed over the interval $\left(\gamma^{-n} x, x\right)$, see for instance [23, Thm. 1.2.5]. Hence,

$$
\begin{aligned}
E_{n}(x) & =\frac{\left(1-\gamma^{-n}\right) x}{\alpha}\left(1+\lambda \int_{y=\gamma^{-n} x}^{x} \frac{E(y)}{\left(1-\gamma^{-n}\right) x} \mathrm{~d} y\right) \\
& =\frac{\left(1-\gamma^{-n}\right) x}{\alpha}+\frac{\lambda}{\alpha} \int_{y=\gamma^{-n} x}^{x} E(y) \mathrm{d} y .
\end{aligned}
$$

Using (1) and (31) we now arrive at

$$
E(x)=\frac{\left(1-Q\left(\gamma^{-1}\right)\right) x}{\alpha}+\frac{\lambda}{\alpha} \sum_{n=1}^{\infty} q_{n} \int_{y=\gamma^{-n} x}^{x} E(y) \mathrm{d} y
$$

Although in the finite-window case $(M<\infty)$ the above integral equation has only meaning for $0<x \leq M$, it is well defined for all $x>0$, and the solution to (33) is unique, see [5]. Define the LT of $E(x)$ :

$$
\hat{e}(\omega):=\int_{x=0}^{\infty} \mathrm{e}^{-\omega x} E(x) \mathrm{d} x .
$$

In [5] it is shown that $\hat{e}(\omega)<\infty$ for $\omega>\lambda / \alpha$. Hence, using that the $q_{n}$ and $E(x)$ are non-negative we may interchange the order of integration and summation (twice), finally arriving at:

$$
\hat{e}(\omega)=\frac{1}{\alpha \omega-\lambda}\left(\frac{1-Q\left(\gamma^{-1}\right)}{\omega}-\lambda \sum_{n=1}^{\infty} q_{n} \hat{e}\left(\gamma^{n} \omega\right)\right) .
$$

Repeated substitution of this equation into itself and applying partial fraction expansion leads us to the following candidate solution:

$$
\hat{e}(\omega)=\frac{1-Q\left(\gamma^{-1}\right)}{\omega} \sum_{i=0}^{\infty} \frac{e_{i}}{\gamma^{i} \alpha \omega-\lambda},
$$

where the $e_{i}$ are constants to be determined. This representation will be justified by showing that it leads us to the unique solutions to (34) and (33). Substituting (35) into (34) and equating the coefficients multiplying the terms $1 /\left(\gamma^{i} \alpha \omega-\lambda\right)$ leads to:

$$
\begin{aligned}
\frac{e_{i}}{e_{0}} & =\frac{1}{1-\gamma^{-i}} \sum_{n=1}^{i} \gamma^{-n} q_{n} \frac{e_{i-n}}{e_{0}}, \quad i \geq 1, \\
e_{0} & =\left(1+\sum_{n=1}^{\infty} \gamma^{-n} q_{n} \sum_{j=0}^{\infty} \frac{e_{j} / e_{0}}{\gamma^{j+n}-1}\right)^{-1} .
\end{aligned}
$$

We note that the ratios $e_{i} / e_{0}$ are non negative and can be computed recursively from (36). Then the normalizing constant $e_{0}>0$ can be computed from (37).

From (36) it can be shown (by induction on $i$ ) that

$$
e_{i} \leq \gamma^{-i} e_{0}, \quad i=1,2, \ldots,
$$

that is, the $e_{i}$ decay exponentially fast in $i$ as $i \rightarrow \infty$. Therefore the right hand side of (35) certainly converges for $\omega>\lambda / \alpha$ and, from its construction, (35) is the solution to (34). By partial fraction (35) can be rewritten as:

$$
\hat{e}(\omega)=\frac{1-Q\left(\gamma^{-1}\right)}{\lambda} \sum_{i=0}^{\infty} e_{i}\left(\frac{1}{\omega-\gamma^{-i} \frac{\lambda}{\alpha}}-\frac{1}{\omega}\right) .
$$

Inverting this LT gives:

$$
E(x)=\frac{1-Q\left(\gamma^{-1}\right)}{\lambda} \sum_{i=0}^{\infty} e_{i}\left(\mathrm{e}^{\gamma^{-i} \frac{\lambda}{\alpha} x}-1\right) .
$$

Using this in (30) we have

$$
P_{M}=\left(1+\left(1-Q\left(\gamma^{-1}\right)\right) \sum_{i=0}^{\infty} e_{i}\left(\mathrm{e}^{\gamma^{-i} \frac{\lambda}{\alpha} M}-1\right)\right)^{-1} \text {. }
$$

Note that because of (38) and

$$
\left(\mathrm{e}^{\gamma^{-i} \frac{\lambda}{\alpha} M}-1\right) \sim \gamma^{-i} \frac{\lambda}{\alpha} M, \quad i \rightarrow \infty,
$$

$P_{M}$ can be computed efficiently from (41).

\section{SPeCial CASE: ONLY ONE CONGESTION SIGNAL PER LOSS EVENT AND A $\gamma=2$}

In this section we specify our results for the important particular case of TCP flow control with only one division of the window by a factor 2 at loss events. Namely, we take $\gamma=2$ and $N \equiv 1\left(q_{1}=1\right.$, and $\left.q_{n}=0, n=2,3, \ldots\right)$ in the model with peak rate limitation, see [15] for a similar model. In Section VII-B we compare the results from this particular case of our model to measurements from the Internet. We worked with long distance connections where congestion signals rarely appear in batches and where the process of loss events is close to 
Poisson [4]. From (14) and (15) we obtain the expressions for the first two moments of the window size distribution.

$$
\begin{gathered}
E[W]=\frac{2 \alpha}{\lambda}\left(1-P_{M}\right) . \\
E\left[W^{2}\right]=\frac{8 \alpha\left[2 \alpha\left(1-P_{M}\right)-\lambda P_{M} M\right]}{3 \lambda^{2}} .
\end{gathered}
$$

The throughput of TCP can be obtained from Equation (11). The distribution function itself or the complementary distribution function $\bar{F}(x)$ is computed successively on the intervals $\left[M / 2^{k}, M / 2^{k-1}\right], k=1,2, \ldots$ using (21) with $\gamma=2$. Recursion (22) reduces to

$$
c_{i+1}^{(k)}=\frac{c_{i}^{(k-1)}}{1-2^{i}}, \quad i=1, \ldots, k-1,
$$

and $c_{1}^{(k)}$ is given by (23). When $M=\infty$, the distribution function is given by (29) with

$$
c_{i}=\frac{1}{1-2^{i}} c_{i-1}, \quad i=1,2, \ldots,
$$

and $c_{0}$ is given by (28).

Finally,

$$
P_{M}=\left(\frac{1}{2} \sum_{i=0}^{\infty} e_{i} \mathrm{e}^{2^{-i}} \frac{\lambda}{\alpha} M\right)^{-1},
$$

where the coefficients $e_{i}$ are given by

$$
\begin{aligned}
\frac{e_{i}}{e_{0}} & =\frac{2^{i-1}}{2^{i}-1} \frac{e_{i-1}}{e_{0}}, \quad i=1,2, \ldots \\
e_{0} & =\left(1+\sum_{i=1}^{\infty} \frac{1}{2^{i}} \frac{e_{i}}{e_{0}}\right)^{-1} .
\end{aligned}
$$

\section{MODEL VALIDATION}

In this section we compare measurements from long distance and long life TCP connections with the results of Section VI ( $N \equiv 1, \gamma=2$, peak rate limitation). Comparison of real measurements with the model with clustered (batch) arrivals of congestion signals is a topic of current research, see also Section VIII.

Due to the large number of hops and the multiplexing of exogenous traffic in network routers, the Poisson loss process assumption is expected to hold on long distance connections [15]. Our TCP receivers implement the delay ACK mechanism [22] and our TCP senders increase their windows in the congestion avoidance mode by approximately one packet every window's worth of ACKs. Thus, we take $\alpha$ equal to 1/(2RTT) [19]. First, we show theoretically how the window size is distributed in the stationary regime. Second, we compare our results to measurements from the Internet.

\section{A. Numerical results}

Consider the case of a long TCP connection with packets of size 1460 bytes and a constant RTT of one second. Using the results of Section VI, we computed the cumulative distribution function $F(x)$ of the window size and its probability density

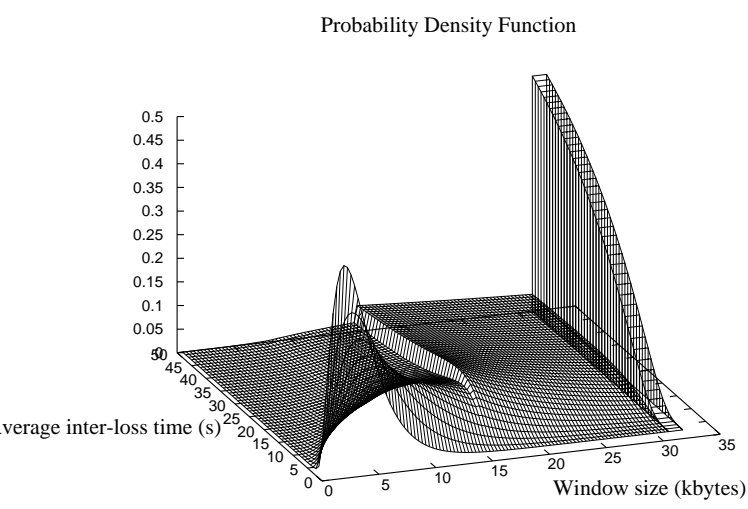

Fig. 3. Limited receiver window: $M=32$ Kbytes Probability Density Function

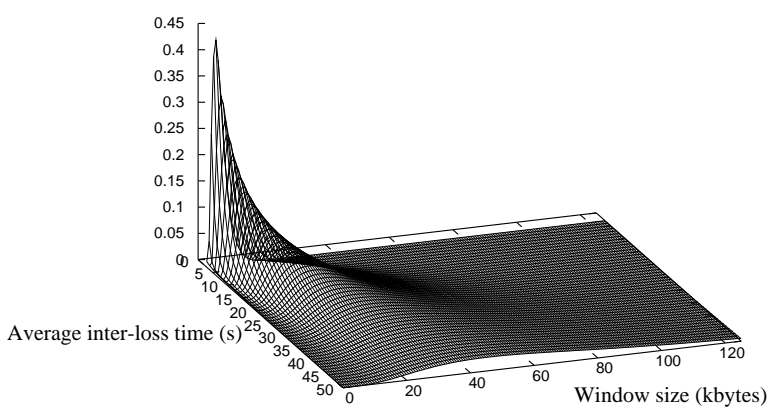

Fig. 4. Unlimited receiver window: $M=\infty$

function $f(x)$ for increasing values of the intensity of losses. We consider the both cases $M=32$ Kbytes and $M=\infty$ (unlimited receiver window). In Figures 3 and 4 we plot our results for the density function $f(x)$. For the case $M=32$ Kbytes, we computed the distribution function successively for the intervals $[M / 2, M],[M / 4, M / 2]$ and so on. In the case of an unlimited receiver window, we used the expression of the density function as an infinite sum of exponentials (Equation (29)). Note that when solving numerically the model for the distribution, several infinite series, e.g. (29) and (42), need to be computed. As we discussed in previous sections, these infinite series converge quite fast; so one needs to use only a small number of terms.

When $M=32$ Kbytes, the discontinuity of $f(x)$ at $x=M / 2$ is clearly seen in Figure 3 (especially for large inter-loss times). The pulse $P_{M}$ at $x=M$ is depicted by an area equal to $P_{M}$. When $M=\infty$, the density function exhibits neither pulses nor discontinuities (Figure 4).

\section{B. Experimental results}

Our experimental testbed consists of a long life and long distance TCP connection between INRIA Sophia Antipolis (France) and Michigan State University (US). The TCP connection is fed at INRIA by an infinite amount of data. The New Reno version of TCP [8] is used for data transfer. We changed the socket buffer at the receiver in order to account for different values of $M$. We considered three values of $M: 32,48$ and 64 Kbytes. For every value of $M$, we ran the TCP connection 


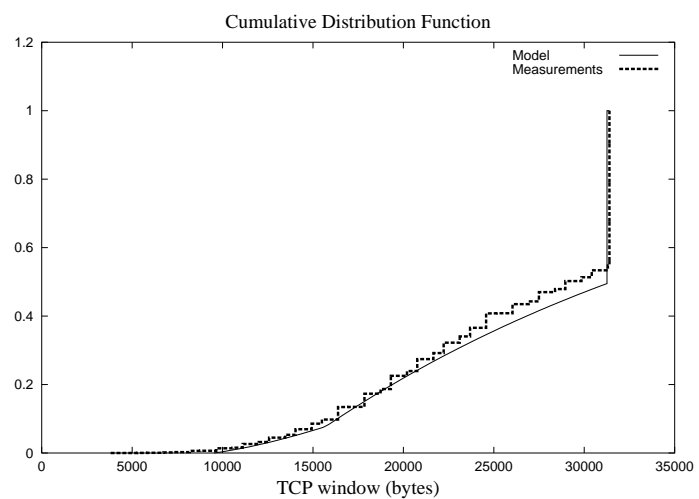

Fig. 5. Receiver window $M=32$ Kbytes

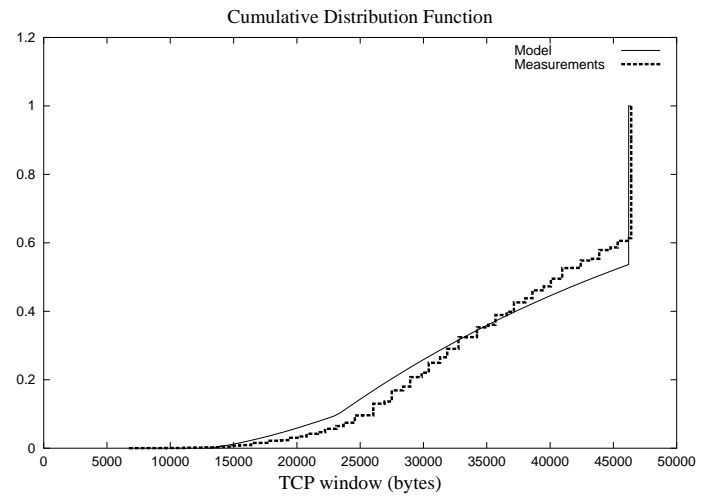

Fig. 6. Receiver window $M=48$ Kbytes

for approximately one hour and we registered the trace of the connection using the tcpdump tool developed at LBNL [13]. We also developed a tool that analyzes the trace of the connection and that detects the times at which the window is reduced. Moreover, our tool gives the average RTT of the connection and the statistics of the window per RTT. We compared for the three values of $M$, the distribution of the window size from measurements to that given by our model. The results are plotted in Figures 5, 6 and 7.

When $M$ is small, we observe a good match between the measured distribution and the one resulting from our model. However, for larger values of $M$, the difference between the two increases. In particular, as $M$ increases, the measured probability density concentrates around the average window size. This deviation can be explained from the measured distribution of times between loss events. First, we plot in Figure 8 the distribution of inter-loss times for $M=32$ Kbytes. This distribution is in agreement with an exponential law, resulting in a good match between the model and the measurements. Figures 9 and 10 show the measured distributions for the other two values of $M$. We observe that the loss process is no longer Poisson, but closer to a deterministic process. Small inter-loss times are less frequent as $M$ increases, and the medium inter-loss times dominate. This results in a degradation of the correspondence between our model and the measurements.

One explanation of the deviation of the loss process from a Poisson process for larger values of $M$ is the following. A true Poisson loss process implies that the time until the next loss event is independent of the past. This is the case when the congestion of the network is dominated by the exogenous traffic and

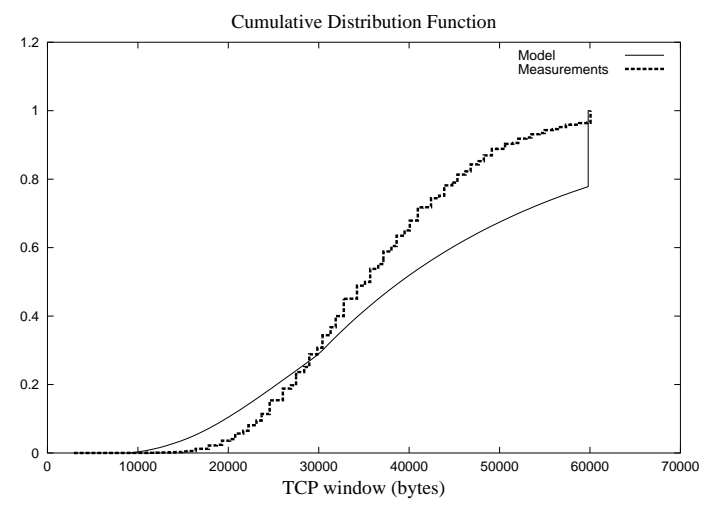

Fig. 7. Receiver window $M=64$ Kbytes

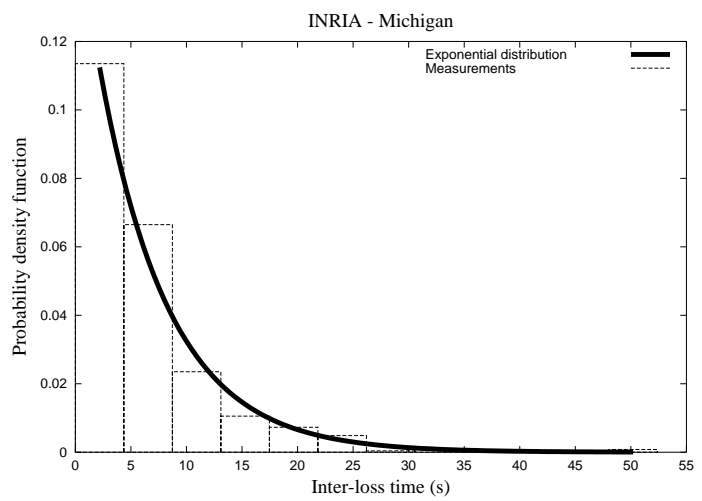

Fig. 8. Case of $M=32$ Kbytes

not dependent on the measured connection. Namely, when the measured connection's share of the available bandwidth on the path is small compared to that of the exogenous traffic. A small $M$ limits the bandwidth share of our connection and limits its impact on the network, resulting in a loss process close to Poisson. However for large $M$, the measured connection realizes a larger share of the bandwidth and thus contributes more to the congestion of network routers. When it reduces its window, the state of the network changes and becomes under-loaded. Some time is needed for the network to be loaded again. This is the reason for which small inter-loss times start to get small probabilities. In such a case when the loss process is close to a deterministic process, a simple fixed-point method as that proposed in [3], [19] can be used to approximate the achieved throughput.

\section{CONCLUSIONS AND FUTURE RESEARCH}

We studied additive-increase multiplicative-decrease flow control mechanisms under the assumption that congestion signals arrive in batches according to a Poisson process. As highlighted in [15], the model can be reformulated as an M/G/1 queuing problem with service time dependent on system workload. We tried to keep the model as general as possible in order to account for a wide range of congestion control strategies. We calculated explicit expressions for the moments as well as the distribution of the transmission rate (of the window size in case of TCP). For the case of single congestion signals, we compared our results to measurements from TCP connections over the Internet. From our experiments, we concluded that our model leads to accurate results when the times between losses are close to being exponentially distributed. 


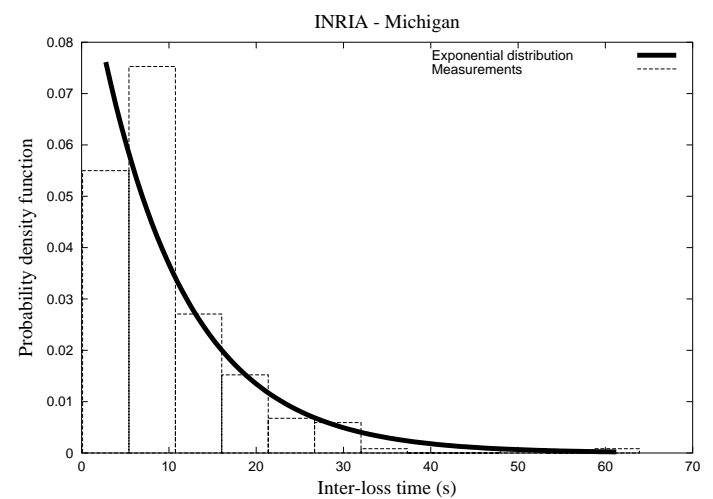

Fig. 9. Case of $M=48$ Kbytes

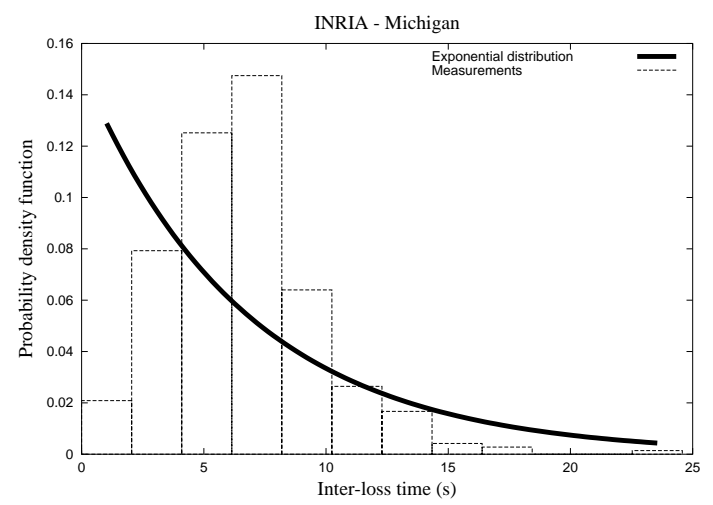

Fig. 10. Case of $M=64$ Kbytes

Currently, we are working on the validation of our model with clustered congestion signals. Our measurements over the Internet have shown that on some paths especially short distance ones, the loss process presents a high degree of burstiness. We are currently also studying the extension of the analysis to more general inter-loss time processes, in particular to MMPPs (Markov Modulated Poisson Processes).

\section{ACKNOWLEDGEMENT}

We would like to thank V. M. Abramov for comments that improved the presentation of the paper. Also, we thank the department of Mathematics of Michigan State University and in particular L.B. Freidovich for providing us with a computer account for our Internet experiments.

\section{A. APPENDIX}

We shall prove here that the infinite series in Equation (28) converges. Denote $a_{i}:=c_{i} / c_{0}$. Using (27), $a_{i}$ satisfies the folowing recurrent relation

$$
a_{i}=\frac{1}{1-\gamma^{i}} \sum_{k=1}^{i} q_{k} a_{i-k}
$$

To prove that series $\sum_{i} a_{i}$ is absolutely convergent, it is enough to prove that the majorant series $\sum_{i} b_{i}$, with $b_{i}$ defined below, is convergent.

$$
b_{i}=\frac{1}{\gamma^{i}-1} \sum_{k=0}^{i-1} b_{k}
$$

Consider $b_{i+1}$

$b_{i+1}=\frac{1}{\gamma^{i+1}-1} \sum_{k=0}^{i} b_{k}=\frac{1}{\gamma^{i+1}-1}\left(\left(\gamma^{i}-1\right) b_{i}+b_{i}\right)=\frac{\gamma_{i}}{\gamma^{i+1}-1} b_{i}$

Thus, $b_{i+1} / b_{i} \rightarrow 1 / \gamma$ as $i \rightarrow \infty$, and therefore, the series $\sum_{i} b_{i}$ (and hence $\sum_{i} a_{i}$ ) is absolutely convergent for $\gamma>1$.

\section{REFERENCES}

[1] The ATM Forum Technical Committee, Traffic Management Specification, Version 4.0, 95-0013R8, Oct. 1995.

[2] V.M. Abramov, "Investigation of a queueing system with service depending on queue length", Donish (pub.), Dushanbe, 1991, (in Russian).

[3] E. Altman, K. Avrachenkov and C. Barakat, "TCP in presence of bursty losses", ACM SIGMETRICS, Jun. 2000.

[4] E. Altman, K. Avrachenkov and C. Barakat, "A stochastic model of TCP/IP with stationary random losses", ACM SIGCOMM, Sep. 2000.

[5] E. Altman, K. Avrachenkov C. Barakat and R. Núñez-Queija, "Statedependent M/G/1 type queueing analysis for congestion control", $C W I$ report PNA-R0005, Jul. 2000.

[6] D. Choi, C. Knessl, C. Tier, "A queueing system with queue length dependent service times, with applications to cell discarding in ATM networks", J. Appl. Math. Stoch. Anal., vol. 12 (1999), no. 1, pp. 35-62.

[7] S. Floyd, "TCP and Explicit Congestion Notification", ACM Computer Communication Review, Vol. 24, No. 5, Oct. 1994, pp. 8-23.

[8] S. Floyd and T. Henderson, "The NewReno Modification to TCP's Fast Recovery Algorithm", RFC 2582, Apr. 1999.

[9] J. Heinanen, T. Finland, F. Baker, W. Weiss, and J. Wroclawski, "Assured Forwarding PHB Group", RFC 2597, Jun. 1999.

[10] V. Jacobson, "Congestion avoidance and control", ACM SIGCOMM, Aug. 1988.

[11] C. Knessl, B.J. Matkowsky, Z. Schuss, and C. Tier, "Asymptotic Analysis of a state-dependent M/G/1 queueing system", SIAM J. Appl. Math., vol. 46 (1986), no. 3, pp. 483-505.

[12] C. Knessl, C. Tier, B.J. Matkowsky, Z. Schuss, "A state-dependent GI/G/1 queue", Eur. J. Appl. Math., vol. 5 (1994), no. 2, pp. 217-241.

[13] LBNL's tcpdump tool, available at http://www-nrg.ee.lbl.gov.

[14] M. Mathis, J. Semke, J. Mahdavi, and T. Ott, "The Macroscopic Behavior of the TCP Congestion Avoidance Algorithm", ACM Computer Сотmunication Review, vol. 27, no. 3, pp. 67-82, Jul. 1997.

[15] V. Misra, W.-B. Gong, D. Towsley, "Stochastic differential equation modeling and analysis of TCP-windowsize behavior", Performance'99, Oct. 1999.

[16] A. Misra, T. Ott, and J. Baras, "The Window Distribution of Multiple TCPs with Random Queues", IEEE GLOBECOM, Dec. 1999.

[17] S.C. Niu, "On queues with dependent interarrival and service times", Naval Res. Logist. Quart., vol. 28 (1981), no. 3, pp. 497-501.

[18] T.J. Ott, J.H.B. Kemperman, and M. Mathis, "The stationary behavior of ideal TCP congestion avoidance", available at: ftp://ftp.telecordia.com/pub/tjo/TCPwindow.ps, Aug. 1996.

[19] J. Padhye, V. Firoiu, D. Towsley, and J. Kurose, "Modeling TCP Throughput: a Simple Model and its Empirical Validation", ACM SIGCOMM, Sep. 1998.

[20] M. Posner," Single-server queues with service time dependent on waiting time", Operations Res., vol. 21 (1973), pp. 610-616.

[21] S. Sahu, D. Towsley, and J. Kurose "A Quantitative Study of Differentiated Services for the Internet" IEEE GLOBECOM, Dec. 1999.

[22] W. Stevens, "TCP Slow Start, Congestion Avoidance, Fast Retransmit, and Fast Recovery Algorithms", RFC 2001, Jan 1997.

[23] H.C. Tijms," Stochastic Models - An Algorithmic Approach", Wiley, Chichester, 1994. 